Copyright 2021 Society of Photo-Optical Instrumentation Engineers (SPIE). One print or electronic copy may be made for personal use only. Systematic reproduction and distribution, duplication of any material in this publication for a fee or for commercial purposes, and modification of the contents of the publication are prohibited.

This is the accepted version of the paper available at https://doi.org/10.1117/12.2594276 


\title{
3D Light Field LED Wall
}

\author{
Tibor Balogh ${ }^{\mathrm{a}}$, Attila Barsi ${ }^{\mathrm{a}}$, Peter A. Kara ${ }^{\mathrm{b}}$, Mary Guindy ${ }^{\mathrm{a}}$, Aniko Simon ${ }^{\mathrm{c}}$, and Zsolt Nagy ${ }^{\mathrm{a}}$ \\ ${ }^{a}$ Holografika, Budapest, Hungary \\ ${ }^{\mathrm{b}}$ Budapest University of Technology and Economics, Budapest, Hungary \\ cSigma Technology, Budapest, Hungary
}

\begin{abstract}
LED technologies expectedly will dominate future displays by outperforming current mainstream technologies, while LED walls have already become the key solution in large-scale displaying. LED walls are not just display walls anymore; they offer a creative modular platform for displaying in any size, aspect, shape, and on any surface. At the time of this paper, there is no real 3D solution for such an attractive technology. In the recent years, rapid miniaturization of LEDs, including microLEDs, and LED control ICs has paved the way for novel LED wall displays with extremely high LED density. In this line, Holografika has developed a unique glasses-free 3D LED wall technology and this article describes the implementation of the 3D LED display prototype, focusing on the challenges in designing the optical elements, and also describes the software components that drive it. To match the high expectations for image quality, the optical system was designed with great care, balancing the conflicting 2D- and 3D-related parameters, like pitch size, angular resolution, FOV, considering practical use cases with typical viewing distances, covering specific LED layouts, up to the theoretical limits with regard to the chip sizes; novel optics for wide angle and high angular resolution beyond what usual lenticular systems can provide; preparing for volume production through designing plastic injection molded optics and tools; custom designed record high-density LED backplane, including PCB, seamlessly tileable LED panels where known slanted structures were not acceptable. The software system was designed to be run on GPUs and to be flexible enough to handle various configurations of display geometry and electronics. Not only are arbitrary layouts supported for the arrangement of the pixels and color channels of the 3D pixel under the sheet array, but also the geometries of the individual cabinets of the LED wall are separately addressable. The software calculates the correct ray field for the light field geometry and the color channel and pixel position shuffling table for the individual cabinets. The rendering consists of two distinct phases. The first phase is rendering the 3D scene for all camera rays. The system can render using various algorithms, such as real-time ray tracing; rasterization with orthogonal projection in the horizontal and perspective projection in the vertical axis, with individual images corresponding to directions selected by the lens array; rasterization of an array of horizontally asymmetric perspective cameras coupled with a ray-selection GPU render pass. The second phase enables shuffling the pixels and color channels to the correct byte order for the LED controller ICs. This allows the system to address individual cabinets. It also allows the usage of different ICs in subsequent generations of the display, without the need to deploy costly FPGAs with large area and power consumption requirements to handle the correct shuffling. Furthermore, the system enables the rendering hardware to scale across multiple PCs and GPUs. This is necessary to allow the system to render the high amount of pixels required to drive the LEDs.
\end{abstract}

Keywords: 3D light field, novel system design, LED, lens array, modular display

Further author information: (Send correspondence to Tibor Balogh)

Tibor Balogh: E-mail: t.balogh@holografika.com

Attila Barsi: E-mail: a.barsi@holografika.com

Peter A. Kara: E-mail: kara@hit.bme.hu

Mary Guindy: E-mail: m.guindy@holografika.com

Aniko Simon: E-mail: aniko.simon@sigmatechnology.se

Zsolt Nagy: E-mail: zs.nagy@holografika.com 


\section{INTRODUCTION}

Based on the scientific knowledge available at the time of this paper and the trends of the recent years, it is most likely that light-emitting diode (LED) technologies shall dominate future displays and that they shall outperform every single mainstream solution of the present day, such as liquid-crystal displays (LCDs) and conventional projection, based on digital light processing (DLP), liquid crystal on silicon (LCoS), and so on. LED walls have become the key solution in the area of large-scale visualization, offering a creative platform for modular, anysize, any-aspect, any-shape and any-surface displaying. Apart from wall-sized visualization, the latest fine-pitch LED walls - with pixel pitches under $1 \mathrm{~mm}$ - shall emerge in the context of home entertainment in the size of conventional televisions. MiniLED-based modular TV sets have already been demonstrated (e.g., Sony Crystal, ${ }^{1}$ Samsung $\mathrm{Wall}^{2}$ ) and the evolving microLED technology paves the way for further size reductions. The recent developments in the miniaturization of LEDs - including microLEDs - and in LED control ICs are the harbinger of novel LED-wall displays with extremely high LED density. While the aforementioned solutions are indeed visually appealing and they resourcefully exploit the opportunities provided by the underlying technologies, it needs to be noted that currently there is still no "real" solution within the context of 3D visualization.

On the other hand, we are approaching the rational limits of $2 \mathrm{D}$ visualization. Going beyond $8 \mathrm{~K}$ ultra-highdefinition (UHD), high-dynamic-range (HDR), high-frame-rate (HFR) and wide-color-gamut (WCG) solutions is not expected to make a significant difference for the average users. Even if we keep embracing objectively better-and-better displays - bringing the reflection of the real world closer to perfection - still, when we are looking at a screen, it is evident that what we see is an artificial image, that is quite different from a natural window-like view.

Therefore, 3D visualization worthy of the real world is still missing; we are lacking solutions that overcome the limitations of the currently existing technologies. Stereoscopic 3D (S3D) glasses-based systems do not offer "real" $3 \mathrm{D}$ experience; they do not provide what the users initially expected and the glasses are rather inconvenient for everyday use. However, the situation is not significantly better for most glasses-free devices, as conventional multiview systems are simply not good enough to show undisturbed 3D visuals.

Stereo LED walls were demonstrated with a polarizing foil on the LED screen, and they were to be observed with the help of polarization glasses. For actual everyday use, however, handing out, collecting and cleaning such glasses at public places is just not practical - it is actually close to impossible to solve efficiently. Glasses-free $3 \mathrm{D}$ video walls were also presented, but these were conventional multiview LCD display panels, with all the well-known multiview artifacts and without the advantages of a modular structure.

The market is waiting for a real solution and major players are already looking for "what's next in 3D"; what will be the next "big thing" in the display industry. The concept of practical 3D light field displays - which was, in fact, pioneered by Holografika ${ }^{3-5}$ - is already acknowledged by the industry and it is considered as the potential candidate for next-generation 3D systems.

Considering the developments of LED technologies, the numerous advantages of modular structures and the capabilities of 3D light field visualization, Holografika developed a unique 3D light field LED wall technology, ${ }^{6}$ solving several technological challenges, such as realizing record-high-density LED backplanes with specific LEDchip layouts - up to the theoretical limits - and developing novel optics for wide angle and high angular resolution, thus enabling the implementation of a seamlessly-scalable 3D light field display.

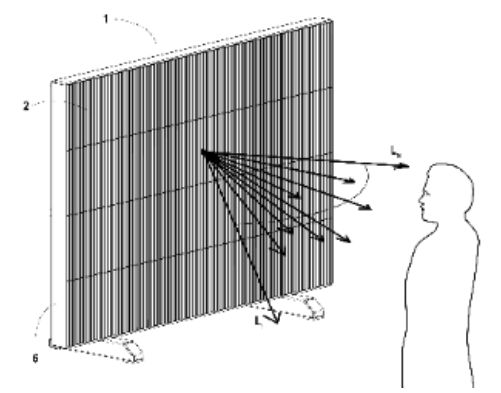

Figure 1. The concept of a 3D light field LED wall. 


\section{THEORETICAL BACKGROUND}

\subsection{Light field technology}

Light field ${ }^{7}$ is a general representation of 3D information that considers a 3D scene as the collection of light rays that are emitted or reflected from 3D scene points. ${ }^{8}$ The 3D light field representation is ideal for 3D displays, since the goal of 3D visualization is to reproduce the light beams from a real or synthetic 3D scene; i.e., emitting light beams to the viewers with the same parameters of direction, position, intensity and color. In other words, the aim is to reconstruct the light field as presented in its natural view.

3D light field visualization represents the next generation of 3D systems that can provide a natural hologramlike 3D view without viewing gears (e.g., glasses) or any other restrictions, opposing other 3D solutions. Light field displays provide various depth cues; not just binocular, but also motion parallax. It means that viewers can look around the objects, which can be behind or even floating in front of the screen. The visualized 3D content can be viewed on a wide viewing range - inside the field of view (FOV) - without any restrictions for the viewers and a continuous view is provided for the entire FOV; there are no invalid zones, neither optical contradiction, nor discomfort. There is no need for positioning or tracking, instead, an unlimited number (i.e., as many as can fit into the viewing area) of freely moving viewers can observe the 3D scene, allowing social experiences and collaborative scenarios with comfortable use.
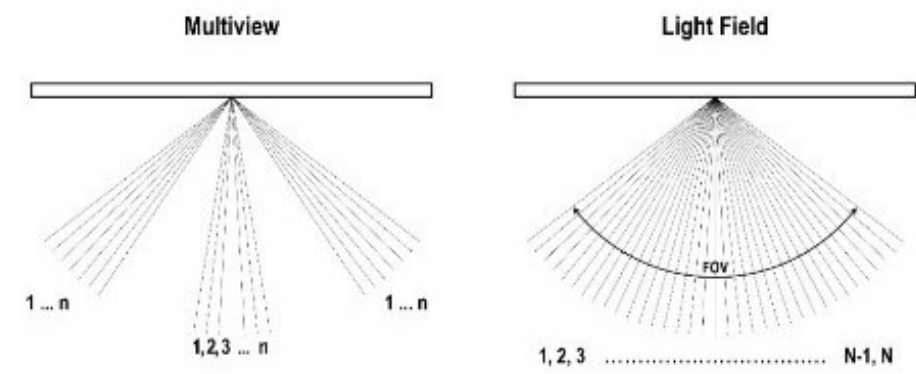

Figure 2. Comparison of the multiview and the light field principle.

In a $3 \mathrm{D}$ image, there is so much more visual information than in a corresponding $2 \mathrm{D}$ one. In order to generate all the light beams forming a high-quality 3D view, 3D displays should have more pixels (spatial multiplexing) or higher speed components (time multiplexing) to deliver this increased optical and processing capacity. It means that while such $3 \mathrm{D}$ displays do provide more views, still, the efficiency of view distribution may be enhanced through certain methods.

Direction-selective light emission is a common feature for any glasses-free 3D system. There is no highlydirectional light emission effect in itself - in case of lasers, see the resonator - so 3D displays always have some optical means, refractive or diffractive optical elements, or optical masks, to deflect light beams. 3D light field displays are optically capable to emit multiple individually controlled light beams from each of their pixels into various directions, as if they were emitted from the points of objects behind screen, or crossing at the points in front of the screen, to display objects floating in mid-air. This light emission characteristic determines the 3D display's capabilities: the FOV (i.e., the freedom given to the viewers); the angular resolution for the depth budget; the pixel size for the resolution and practical screen dimensions.

\subsection{Relevant challenges}

Besides the challenge for high pixel counts in 3D visualization, the "game" is about the pixel size; how to make the smallest 3D pixels - as in similar-sized conventional 2D systems - how to arrange a large number of emitters over the surface of the screen, in order to achieve record-high densities. These are indeed crucial problems to be solved for LED-wall-type systems. One may wonder whether it is even possible to build a practical glasses-free 3D light field LED wall based on the available state-of-the-art LED components. Due to these considerations, the basic conflicting parameters should be examined and optimized for both $2 \mathrm{D}$ and $3 \mathrm{D}$ performance. 


\subsubsection{D quality}

If the viewer is too close to the display, the eye can resolve the pixels (i.e., the individual LEDs), and the display looks rather pixelated. In terms of $2 \mathrm{D}$ quality, there is a minimum viewing distance, at which the visualized content still appears to be adequate. As a subjective rule of thumb at LED walls of various pitch sizes, the acceptable optimal viewing distance in meters is approximately equal to the pixel size in millimeters.

$$
\text { ViewingDistance } \geq 1000 \times \text { PixelSize }
$$

\subsubsection{D quality}

In case of light field visualization, there are a distinct number of views divided across the FOV. The angular difference between the view directions is the angular resolution. Angular resolution is without a doubt the primary parameter of 3D quality; smaller angular resolution values result in better (i.e., deeper) 3D.

3D sensation is created by addressing the two eyes of the observer by different (i.e., differently colored) light beams emitted from the same point on the screen. Physically, the angular resolution is the angular distance between the closest independent light beams. These light beams diverge with the viewing distance. At a specific viewing distance, the width of the viewing zone exceeds the $65 \mathrm{~mm}$ interpupilar distance. Beyond that distance, both eyes can be in the same viewing zone, seeing the same color on the display and thus they are not addressed by distinct light beams. There is no strict limit, however, 3D information is not necessarily lost, since the motion parallax is still relevant, and particularly in case of LED wall utilization of the technology, viewers typically tend to move from one position to another. Still, exceeding this distance can lead to degradations in perceived 3D quality. Therefore, in terms of 3D quality, there is a maximum viewing distance.

$$
\text { ViewingDistance } \leq \frac{65[\mathrm{~mm}]}{\tan (\text { AngularResolution })}
$$

The precise meaning of this aforementioned maximum viewing distance in the context of 3D sensation was systematically investigated in the work of Kara et al. ${ }^{9}$ The obtained results clearly indicate that viewers positioned at this distance are unable to decide between the $2 \mathrm{D}$ or $3 \mathrm{D}$ visual nature of the content. There are statistically significant differences between the selected viewing positions, and a near-linear relationship can be modeled between the viewing distance and 3D sensation. However, these findings only apply to still (i.e., not moving) observers, as sideways motion may compensate this loss. ${ }^{10,11}$

One can add two more equations to connect the physical quantities of Equation 1 and 2.

$$
\text { NumberOfViews } \times \text { ChipSize }=\text { PixelSize }
$$

$$
\text { NumberOfViews } \times \text { AngularResolution }=F O V
$$

Combining all the equations above leads to an important result. To keep both 2D and 3D quality in balance, the following equation should be considered:

$$
\text { FOV } \times \text { ChipSize } \leq 3.73[\mathrm{deg} \times \mathrm{mm}]
$$

The equation clearly states that the chip size must be minimized against the FOV. The chip size is limited by the existing technologies, while applications typically require wider FOV.

The pixels of LED walls are realized as single RGB SMD packages, or $4 \times 1$ packages for finer pitches. Though RGB SMD LED packages are available under $1 \mathrm{~mm} \times 1 \mathrm{~mm}$ (0404, 0202 parts), this cannot be an approach for a functioning 3D concept. In order to display true 3D images, an additional order of magnitude of light emitters is needed in comparison to 2D solutions, which is a notable challenge for LED displays - having a packaged LED or LED chip as a sub-pixel. The pitch size of a 3D display and the number of light beams to be emitted from the pixel pitch are conflicting parameters. 
We introduced a novel arrangement decoupled from the package-based constraints. We examined the actual light emission of LED chips to reach the theoretical maximum in achieving the highest optical density arrangement. Therefore, we could set up a concept with practical parameters, such as a 5-mm pitch, a wide 70-degree FOV and an angular resolution below 2 degrees.

Horizontal parallax in this context - and quite frankly, in general as well - is more important than vertical parallax. Our eyes are displaced horizontally and we move horizontally in front of such screens, not to mention that the majority of the movements in our daily lives is horizontal. As such, distributing the light asymmetrically from pixels into horizontal directions only can significantly reduce the number of necessary light beams (e.g., it is enough to emit 50 light beams from a given pixel instead of $50 \times 50$ for a slight visual gain). Optimizing on the industrial trinity of complexity, costs and performance, horizontal-only parallax (HOP) systems represent the only viable option for the coming years.

Another challenge was the optics. Single-element lenticular sheets applied in conventional glasses-free 3D displays can give satisfactory optical quality up to 41-42 degrees. For a commercially viable 3D LED wall, however, considering the potential public use cases, much wider FOV is expected without any repeated views or invalid zones. We designed a completely new multilayer optical system that provides a 72 -degree FOV with excellent resolution. The optical layers were designed for compactness and mass production, suitable for plastic injection molding, and possibly "future-proof", even for the upcoming smaller LED chips.

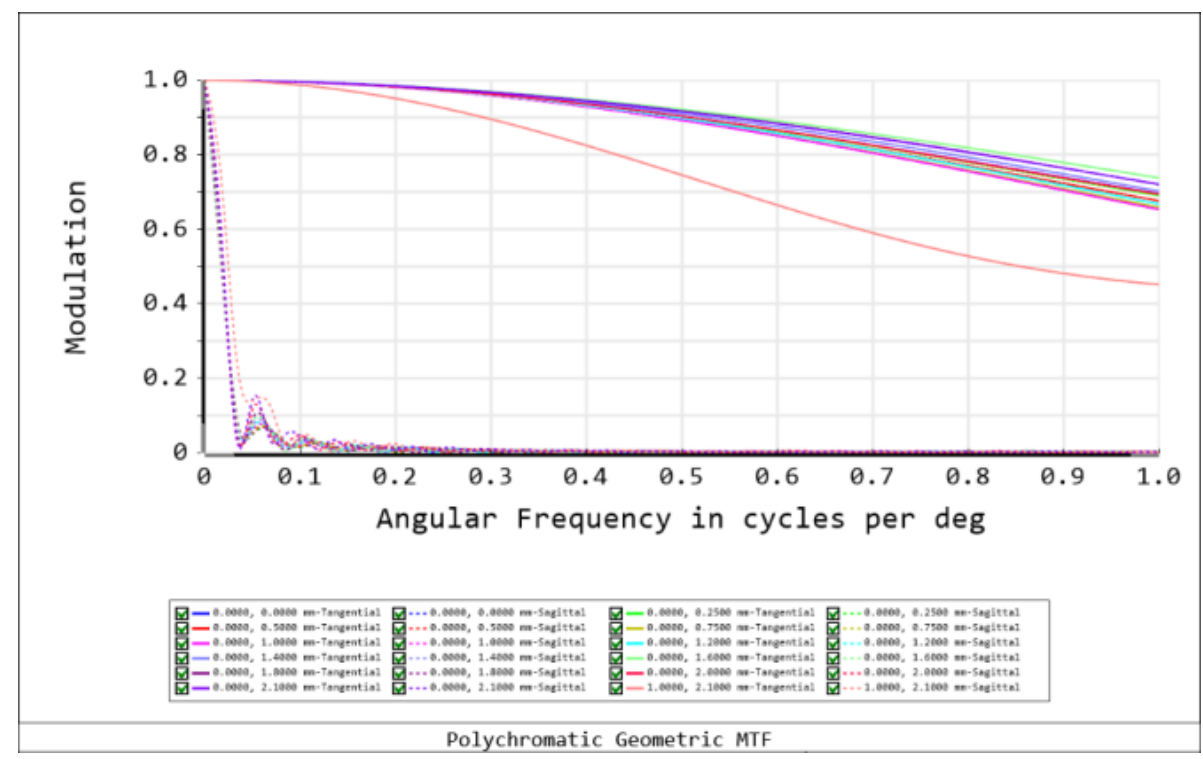

Figure 3. The MTF performance of the new multilayer optics.

It is a common approach at single-panel 3D screens to apply slanted lenticulars or parallax barriers to compensate the horizontal versus vertical resolution loss with the number of views. For LED wall modular structures, however, only properly oriented periodic features - allowing seamless tiling - are applicable, that otherwise would be impossible with slanted structures, leading to the fractions of optical elements.

The modular approach is the effective way to build systems with very high pixel counts. In case of LED walls, the resolution evidently scales with the size. As of the general challenges of extremely high LED densities, the modular approach not only removes the technological barriers, but it can also be an economic point in the production yield or a practical point regarding maintenance (i.e., it makes it easy to replace broken components).

\section{HARDWARE CONSIDERATIONS AND IMPLEMENTATION}

LED chips, being emissive components, are ideal in display technologies: they are efficient, enabling high brightness at reasonable power consumption; they provide unprecedented contrast with real black levels (i.e., when turned off); they offer wide color gamut, close to laser emitters, but on slightly wider spectrum, eluding speckle; and are quite reliable, due to their long lifetime. 


\subsection{LED backplane}

The proprietary custom LED backplane is the most critical component within the 3D LED wall. The development and manufacturing of the high-density LED backplane was a real technological challenge. During the phase of design, the printed circuit board (PCB) wiring was a demanding task, since particularly on the front side, there is no space left between the densely arranged LEDs for the large number of connections other than the necessary clearances. The extreme LED density results in strict requirements on the PCB substrate as well, and the complex multilayer PCB is close to the limits of manufacturing feasibility.
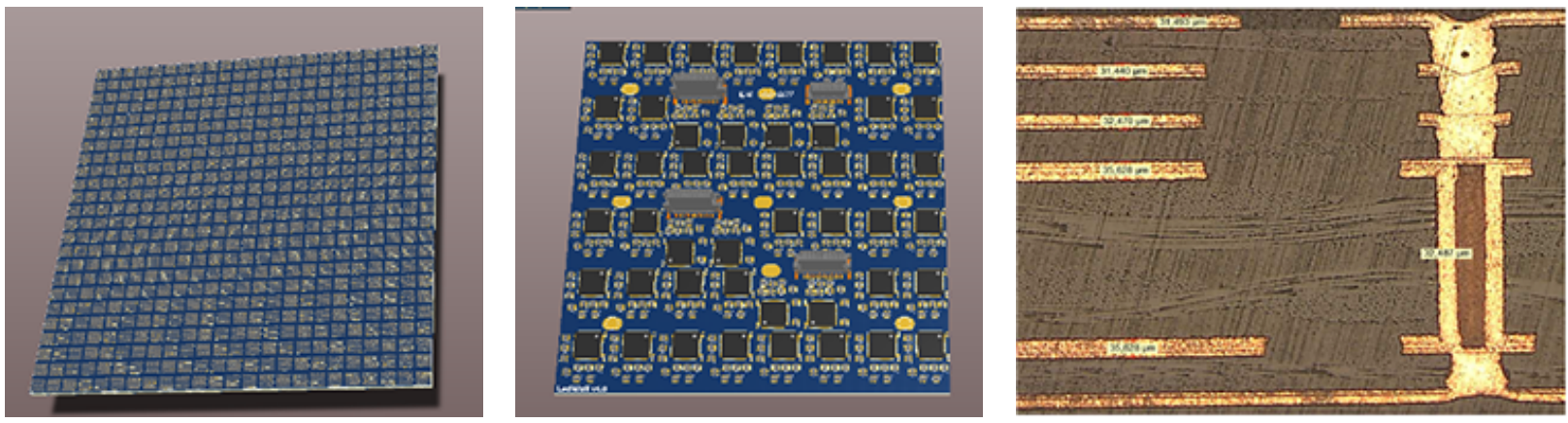

Figure 4. The final LED board with a cross section of the PCB.

\subsection{Multilayer optics}

The plastic optics were prototyped, tested, and with iterations, the parameters were fine-tuned for cost-effective mass manufacturing. At the injection moulding process, the optical inserts, the mould design was in focus, to provide a proper temperature profile for the flow of the melted optical material. Then the optimum cooling distribution was critical, in order to avoid any deformation or significant shrinking, so that the parts of the complete system were in the range of tolerance.

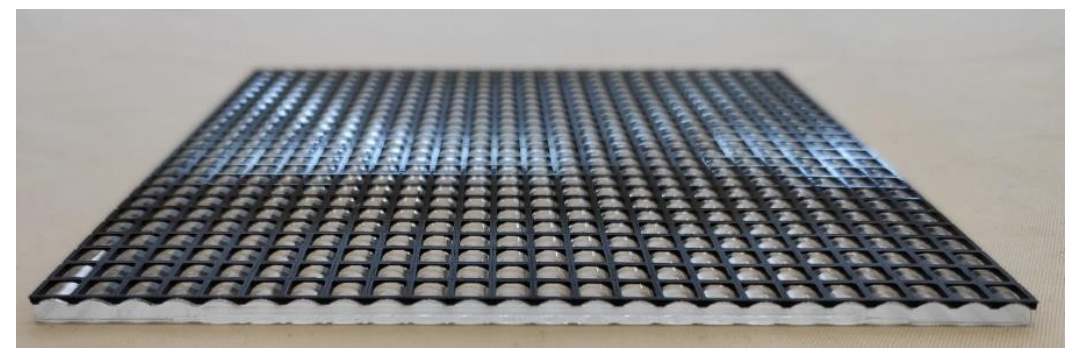

Figure 5. The plastic optics.

Similarly, regarding integration, bonding the optics to the LED backplane was a delicate design and implementation task, with proper substrate and material choices, shapes, bonding processes to avoid thermal stress and damages during operation.

\subsection{Mechanics}

The role of the mechanics was not only to precisely match and hold the modules together, but also to provide cooling. A compact yet rather structured mechanics was designed from scratch, coming altogether in a thickness of $35 \mathrm{~mm}$. Four panels together form a mechanical unit called cabinet. The cabinet has a mechanical holder frame and this is the basic building block for the 3D LED wall. The cooling is solved through the back side of the LED board. The heat generated by the LEDs is transferred to the back side of the PCB by the large number of vias. While it caused a difficulty at the stage of manufacturing, it now helps distributing the heat within the LED board. Heat-conductive layers are used to transfer the heat to the mechanics. We made simulations of the thermal behavior of the system for the various mechanical shapes and dimensions that were in good agreement with the measured data. Therefore, passive cooling was proven to be satisfactory in terms of heat dissipation, even when the LEDs are pushed hard for high levels of brightness. 


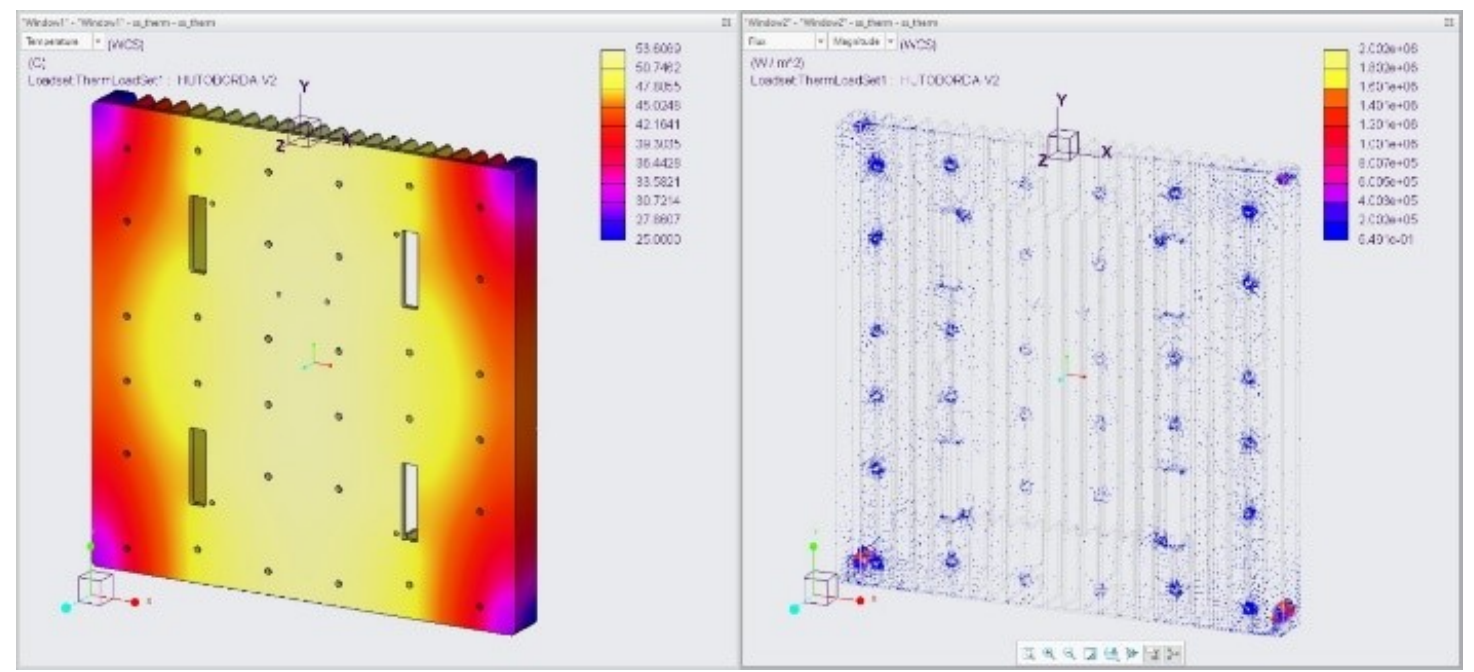

Figure 6. Thermal simulation of the mechanics.

\subsection{Assembly, tests and measurements}

With all the components manufactured and having all subsystems ready - including the driving and FPGAbased control electronics - it was also a considerable development effort to assemble the prototype panels and to perform operational tests on them.

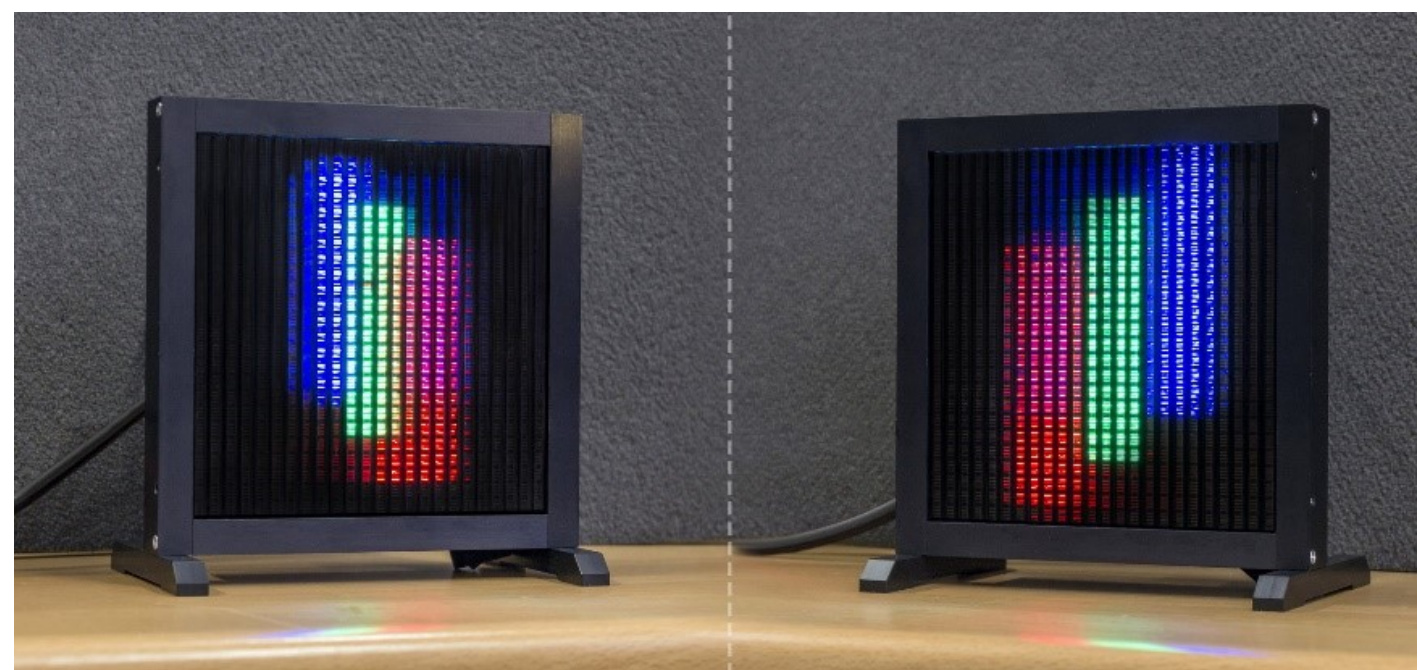

Figure 7. Close-up of the prototype panel from left and right.

After the functional testing, measurements were performed. All of the measurements were done at $3.75 \mathrm{~mA}$ current settings for the LEDs, which is $75 \%$ of the $5 \mathrm{~mA}$ maximum current. The results of these measurements are summarized in Table 1.

\begin{tabular}{|l|l|}
\hline Average brightness of the white screen & LWavg $=1882 \mathrm{~cd} / \mathrm{m}^{2}$ \\
\hline Average brightness of the black screen & $\mathrm{LBavg}=0.03 \mathrm{~cd} / \mathrm{m}^{2}$ \\
\hline Luminance contrast & $\mathrm{C}=\mathrm{LWavg} / \mathrm{LBavg}=1882 / 0.03=\sim 60000$ \\
\hline
\end{tabular}

Table 1. Brightness and contrast values.

Color measurements were done with white and with the RGB primary colors. Color uniformity was evaluated through a nine-point measurement, similarly to the evaluation of brightness uniformity. 

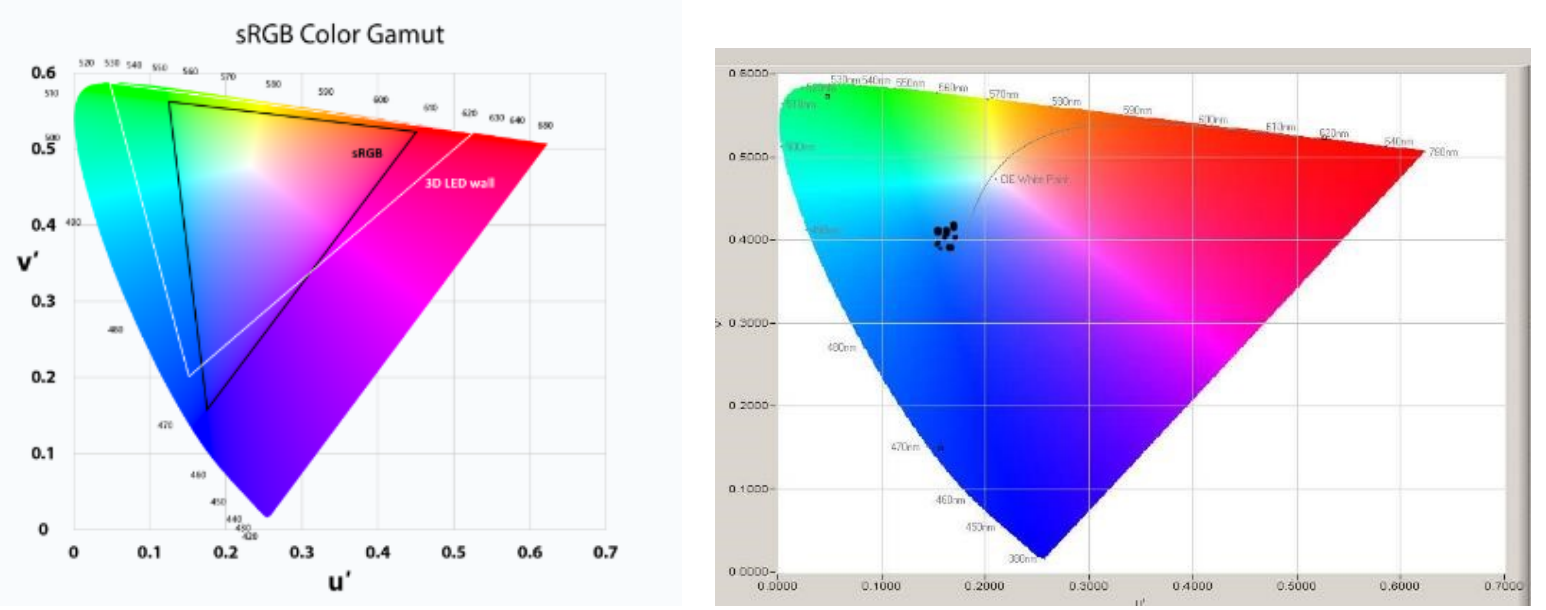

Figure 8. Results of the color gamut and color uniformity measurements.

Crosstalk was measured in a turntable setup with the help of a light measurement device. The measurement was performed for the 3 primary colors. As the red chip is the smallest in the package, we expected the best (i.e., lowest) crosstalk for the red color, and somewhat higher crosstalk values for green and blue. During the measurement, alternating black and full-color views were displayed and the screen brightness was measured as the function of the viewing angle relative to the perpendicular plane. Crosstalk values represent the ratio of the neighboring valleys to peaks. The best crosstalk is measured for the red color - as expected - down to $8 \%$ of crosstalk values. The blue and green values are higher, approximately $12 \%$ and $15 \%$, respectively.

\subsection{D light field LED wall prototype}

With the cabinets completed, each of the 6 cabinets, $240 \mathrm{~mm} \times 240 \mathrm{~mm}$ in size, was assembled to a $720 \mathrm{~mm}$ $\times 480 \mathrm{~mm}$ demonstrator, building the first ever 3D light field LED wall prototype. There parameters of the prototype are summarized in Table 2.

\begin{tabular}{|l|l|}
\hline Demonstrator & Parameters \\
\hline Pixel size & $5 \times 5 \mathrm{~mm}$ \\
\hline Panel size & $120 \times 120 \mathrm{~mm}$ \\
\hline 2D equivalent resolution of a panel $(\mathrm{H} \times \mathrm{V})$ & $24 \times 24 \mathrm{px}$ \\
\hline Cabinet size & $240 \times 240 \mathrm{~mm}$ \\
\hline LED forward voltages $(\mathrm{R} / \mathrm{G} / \mathrm{B})$ & $2.0 \mathrm{~V} / 3.3 \mathrm{~V} / 3.3 \mathrm{~V}$ \\
\hline Max LED current & $5 \mathrm{~mA}$ \\
\hline Total current per panel & $\max 13 \mathrm{~A}$ \\
\hline Power consumption & $\max 3.4 \mathrm{~kW} / \mathrm{m}^{2}$ \\
\hline Brightness at half current & $1000 \mathrm{~cd} / \mathrm{m}^{2}$ \\
\hline Brightness at $75 \%$ current & $1800 \mathrm{~cd} / \mathrm{m}^{2}$ \\
\hline Field of view & 70 degrees \\
\hline Angular resolution & 1.9 degrees \\
\hline Max frame rate & 75 frame $/ \mathrm{sec} @ 25 \mathrm{MHz}$ \\
& 60 frame $/ \mathrm{sec} @ 20 \mathrm{MHz}$ \\
\hline Cooling & Passive \\
\hline Demonstrator size -6 cabinets & $720 \mathrm{x} 480 \mathrm{x} 35 \mathrm{~mm}$ \\
\hline
\end{tabular}

Table 2. Parameters of the prototype. 


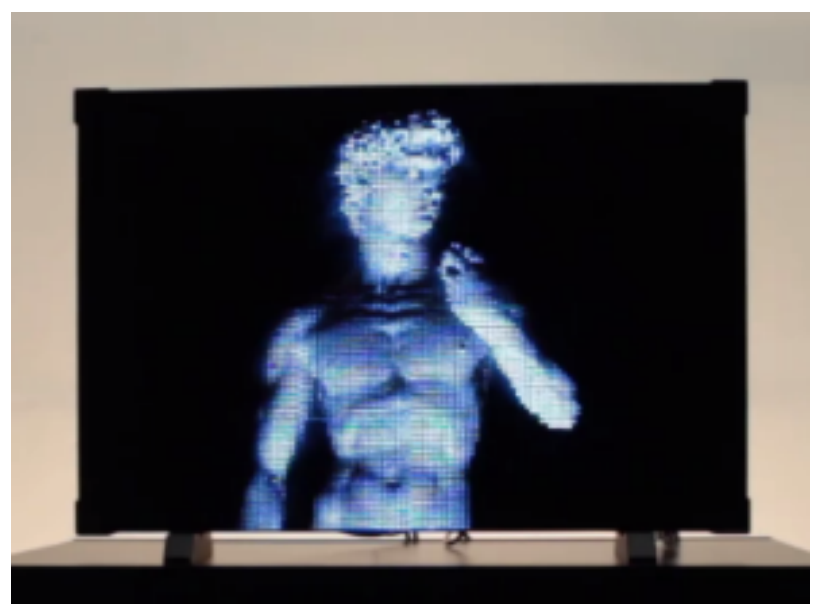

Figure 9. The 3D light field LED wall in operation.

\section{SOFTWARE CONSIDERATIONS AND IMPLEMENTATION}

While designing the rendering algorithms for the display, a distributed, real-time rendering system was envisioned, that can address the cabinets of the display individually. To achieve this goal, rendering and pixel shuffling for the 3D LED Wall was implemented on an existing C++ library stack developed for light field displays by Holografika, containing the HoloSDK API and the ClusteredRenderer rendering engine.

\subsection{Phase 1: Rendering the camera rays}

The following real-time light field rendering algorithms have been developed for the display:

- Rasterization using orthogonal projection horizontally and perspective projection vertically, followed by a resampling of the results using a simple texture mapping approach during the reshuffling phase to compensate for the perspective differences of individual pixel columns of the screen.

This algorithm assigns a camera and generates an image per emitted direction. All camera directions are selected to be the inverse of the respective emitted direction. The horizontal perspective is orthographic. The vertical perspective is calculated by projecting the top and the bottom pixel row onto the orthographic projection's near clipping plane. The FOV corresponding to the maximum projected distance between a column's top and bottom pixel is selected to be the FOV. The resolution is increased so that the resolution of the column with the smallest projected distance between its top and bottom pixel has full vertical resolution during resampling. Stencil buffers or masks can help during the rasterization phase to skip the evaluation of unused pixels.

This rasterization technique uses per column precalculated viewing positions for correct light field per pixel lighting of virtual objects. Images are typically rendered into a $2 \mathrm{D}$ texture array (the maximum of the required vertical resolutions is taken as the common image resolution). This helps with the next phase, where these images are resampled and shuffled to achieve the correct byte order for the display's input.

- Rasterization using sampling cameras that are asymmetrically perspective in the horizontal direction. In this case, a section of the observer line within the range of the display's FOV is sampled uniformly to select camera eye positions.

All camera directions are selected to be perpendicular to the screen. For each camera, the vertical perspective is set so that the camera frustum's top and bottom planes include the top and the bottom side (segment) of the display respectively. The left and the right parameters of the horizontal perspective are set asymmetrically so that the left and the right planes of the camera's frustum include the left and the right side (segment) of the display respectively. These images are resampled to get the correct image for the display and submitted to the byte order correction phase. Lighting algorithms do not need to be changed from the traditional $2 \mathrm{D}$ rendering pipeline. 
Please note that this technique allows some low cost rendering techniques, such as vertex lighting, however, some image space techniques (especially the ones involving fixed kernel sizes) might not behave as expected due to the resampling phase. This technique was mainly used to provide compatibility with engines that are not light field aware, such as Holografika's plugin module for Unity*.

- Modern GPU hardware also allows for rendering implementations using ray tracing algorithms. We have implemented ray tracing using both compute shaders ${ }^{12}$ and NVIDIA OptiX ${ }^{\dagger}$. The advantages of these techniques include that they allow an exact evaluation of the light field (there is no need for any resampling). Lighting is also automatically calculated correctly. When used on a display, where only the pixel positions and/or the color channels are rearranged, it also allows for a rendering that produces the correct image in a single pass, thus reducing the need for expensive memory read/write operations on the GPU.
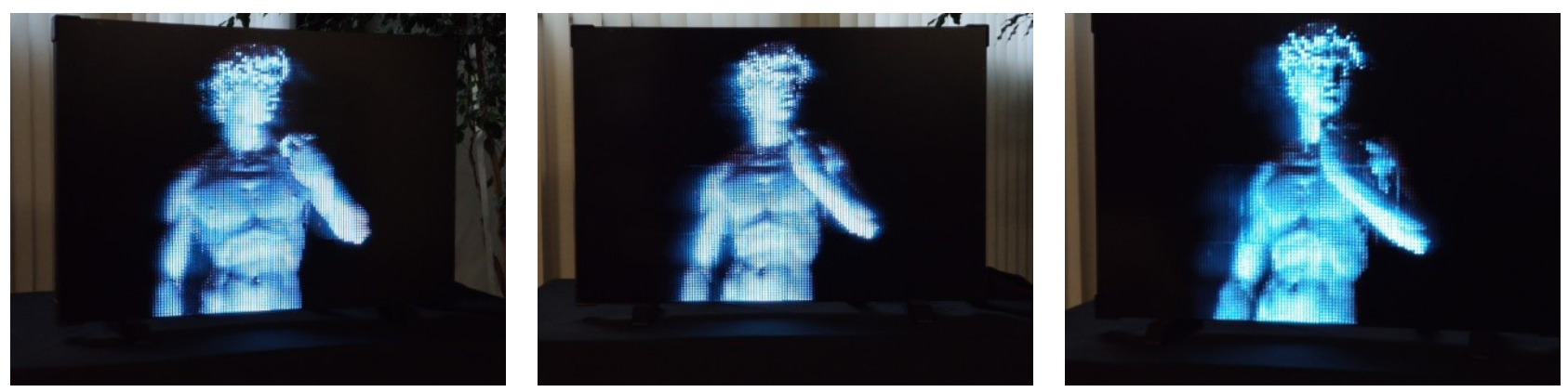

Figure 10. Ray-traced content on the 3D light field LED wall, as seen from different directions.

\subsection{Phase 2: Transmission in the correct byte order}

The correct byte order can be configured via a flexible file format and a lookup table is precalculated if necessary. This lookup table reorders the image set into the final byte array that is output via the HDMI port of the graphics card. This phase can be skipped in case of ray tracing and FPGA support for the grouping of bytes (R,G,B channel in arbitrary, but fixed channel order) corresponding to a single pixel.

\section{CONCLUSION}

Holografika developed the world's first ever 3D Light Field LED Wall system, that represents a new product category in a multi-billion-dollar market. The first 5-mm-pitch prototype cabinets are $240 \mathrm{~mm} \times 240 \mathrm{~mm}$ seamlessly-tileable modules with a newly developed dedicated control system. The 3D light field LED wall shows natural glasses-free 3D content on a wide FOV, providing a totally novel user experience. Newer generation panels are under development and further industrialization is needed for mass production, however for early adapters the system is already close to the market.

The modular character of the light field LED wall enables the attractive option to build any-size, any-aspect and any-shape 3D displays. Relative to the size, the 3D LED wall modules can be considered a flat panel; a lightweight solution that can practically match several use cases, like indoor and outdoor displays at public spaces, visually impressive concerts and stage technologies, TV studios, etc. The utilization scenarios of the light field LED wall include both permanent and easy-to-build temporary installations as well.

The worldwide LED video display market achieved spectacular growth in 2019, with a total revenue rising by $34.7 \%$. The global LED video display market revenue totaled $\$ 5.7$ billion in 2019 , up from $\$ 4.2$ billion in 2018 , according to the Omdia LED Video Displays Intelligence Service. ${ }^{13}$

There are various forecasts with diverse numbers and categories, that might be influenced by the current pandemic, but according to the original trends, the global LED display market will reach $\$ 9,4$ billion by 2022 ,

\footnotetext{
*https://unity.com/

${ }^{\dagger}$ https://developer.nvidia.com/optix
} 
according to LED Inside, ${ }^{14}$ and even more optimistic forecasts were also published, like $\$ 22.4$ billion by 2024 (with $26 \%$ CAGR), up to $\$ 73.5$ billion by 2025 (with 35\% CAGR), according to Research and Markets. ${ }^{15}$

Summa summarum, there is an evident window for a novel product category on the market.

\section{Acknowledgments}

This project was financed by 2018-2.1.3-EUREKA-2018-00007 from the NRDI fund, Hungary. The work presented in this paper was also supported by the European Union's Horizon 2020 research and innovation programme under the Marie Skłodowska-Curie grant agreement No 813170.

\section{REFERENCES}

[1] Mertens, R., "Sony demonstrate two Crystal-LED displays at ISE 2018," (2018). https://www.microled-info.com/sony-demonstrate-two-crystal-led-displays-ise-2018.

[2] Mertens, R., "Two out of three SID Displays of Year Awards go to Micro-LED displays," (2018). https://www.microled-info.com/two-out-three-sid-displays-year-awards-go-micro-led-displays.

[3] Balogh, T., "The HoloVizio system," in [Stereoscopic Displays and Virtual Reality Systems XIII], SPIE (2006).

[4] Balogh, T., Kovács, P. T., and Barsi, A., "Holovizio 3D display system," in [3DTV Conference], IEEE (2007).

[5] Balogh, T., "HoloVizio: The Light Field Display System," in [International Workshop on 3D Information Technology (3DIT)], (2007).

[6] Balogh, T., "3D light field LED-wall display," (2018). Patent WO2019/155243 (PCT/HU2018/000005), assignee: Holografika.

[7] Lippmann, G., "Epreuves reversibles photographies integrals," Comptes-Rendus Academie des Sciences 146, 446-451 (1908).

[8] Gershun, A., "The light field," Journal of Mathematics and Physics 18(1-4), 51-151 (1939).

[9] Kara, P. A., Tamboli, R. R., Cserkaszky, A., Barsi, A., Simon, A., Kusz, A., Bokor, L., and Martini, M. G., "Objective and subjective assessment of binocular disparity for projection-based light field displays," in [International Conference on 3D Immersion (IC3D)], IEEE (2019).

[10] Kara, P. A., Cserkaszky, A., Barsi, A., Papp, T., Martini, M. G., and Bokor, L., "The Interdependence of Spatial and Angular Resolution in the Quality of Experience of Light Field Visualization," in [International Conference on 3D Immersion (IC3D)], IEEE (2017).

[11] Kara, P. A., Tamboli, R. R., Cserkaszky, A., Martini, M. G., Barsi, A., and Bokor, L., "The Viewing Conditions of Light-Field Video for Subjective Quality Assessment," in [International Conference on 3D Immersion (IC3D)], IEEE (2018).

[12] Doronin, O., Barsi, A., Kara, P. A., and Martini, M. G., "Ray tracing for holovizio light field displays," in [International Conference on 3D Immersion (IC3D)], IEEE (2017).

[13] Park, K. and Badier, J.-A., "Global LED video display market revenues reach $\$ 1.8$ billion in Q4 2019 and record 34.7 percent year-over-year growth in 2019," (2020). https://omdia.tech.informa.com/OM006242/Global-LED-video-display-market-revenues-reach-18-billionin-Q4-2019-and-record-347-percent-yearoveryear-growth-in-2019.

[14] Wu, B., "LEDinside 2020 Global LED Video Wall Market Outlook- Meeting Room, Sales Channel and Price Trend," (2019). https://www.ledinside.com/node/30746.

[15] Research and Markets, "Global LED Video Walls Market Size, Market Share, Application Analysis, Regional Outlook, Growth Trends, Key Players, Competitive Strategies and Forecasts, 2017 to 2025," (2017). hhttps://www.researchandmarkets.com/research/gt8v65/global_led_video?w=4. 